\title{
AN ADAPTIVE ALGORITHM TO ACCELERATE MULTI-PARAMETER MONTE CARLO COMPUTATIONS*
}

\author{
BY \\ DONALD L. HITZL AND FRANK ZELE \\ Lockheed Palo Alto Research Laboratory
}

\begin{abstract}
Estimator expansions in terms of orthonormal Hermite polynomials show particular promise for variance reduction in Monte Carlo computer simulations. This paper briefly describes some new adaptive refinements to the basic variance reduction procedure so that the acceleration towards convergence of multi-parameter Monte Carlo computations is further improved. Simulation results using the adaptive algorithm are presented for a five-parameter model problem.
\end{abstract}

Introduction. The point of view taken in this paper is to regard Monte Carlo computations as an approximate form of numerical integration [1] since, for multi-parameter problems ( 25 to 10 random variables for example), the necessary integrals describing the stochastic system can rarely be evaluated either in closed form or by conventional numerical integration routines. Monte Carlo methods are then applied quite often to high-dimensional problems and, as a result, techniques for variance reduction (or, equivalently, convergence acceleration) are of considerable interest.

A rather promising variance reduction procedure was first proposed by Chorin [2] in 1971 for problems with one random variable. The technique is based upon constructing approximations to the desired estimate in terms of orthonormal Hermite polynomial series expansions. The basic Chorin approach has now been extended and refined by the present authors to include:

(i) multi-parameter problems [3];

(ii) use of so-called equidistributed random numbers [3];

(iii) symmetrizing the original Chorin estimate $C$ and obtaining the symmetric Chorin $(S C)$ and trisymmetric Chorin (TSC) estimates by averaging [3, 4, 5]. In this way the available data is used more effectively; it is shown in [5] that the TSC estimate yields a universally smaller Monte Carlo error variance than the basic $C$ estimate;

(iv) an adaptive series selection algorithm $[4,5]$ so that only those coefficients determined with sufficient precision are retained in the Hermite polynomial expansion of the estimator;

* Received April 28, 1980. It is a pleasure to thank Mr. T. J. Ross of the Missile Systems Division of LMSC for his long-term interest and support of this research. The authors are also deeply grateful to Mr. F. H. Maltz (presently at the Naval Underwater Systems Center, New London, CT) for his collaboration in all phases of this work from 1975 to 1979 . Finally, we wish to acknowledge Miss S. R. Wishmire and Ms. G. K. Wu for their efforts in typing the manuscript. 
(v) a new adaptive refinement so that the tolerance level (Tol) for acceptance or rejection of an individual term in the estimator expansion is continuously adjusted for each value of $\mathrm{N}$ (the number of sample trajectories) used in the computations.

The purpose of this paper is to briefly review the symmetrized Chorin estimators, to describe the adaptive procedures currently in use, and to illustrate all aspects of this method with simulation results from a one-dimensional model problem for a ballistic reentry trajectory having random perturbations in the dynamics. For this so-called "bullet problem", initial results with only the $C$ estimator appear in [3, pp. 358-365]; highly refined estimates obtained using the adaptive symmetrized procedures are presented here.

Finally, it should be emphasized that these advanced estimators were developed originally for application to multi-parameter trajectory dispersion studies. In particular, estimates of increased precision were desired for such quantities as range, impact point, and dispersion velocities. The present version of the computer program [6] has been applied to boost, deployment, and reentry trajectory simulations involving up to 40 random input parameters.

Accelerated estimators. A wide variety of methods has been developed for reducing the error variance in Monte Carlo computations. The specific technique to be described here belongs to the general class of methods called control variate.

The ordinary (or direct D) Monte Carlo estimate is defined by

$$
\hat{f}=\frac{1}{N}\left(f_{1}+f_{2}+\cdots+f_{N}\right)=\frac{1}{N} \sum_{1}^{N} f_{i}
$$

where the function $f_{i}=f\left(\mathbf{x}_{i}\right)$ depends upon a Gauss vector $\mathbf{x}$ of $p$ independent components

$$
\mathbf{x}^{T}=[x(1) x(2) \ldots x(p)]
$$

all of which have mean zero and variance one. The control variate estimate is given by

$$
\hat{f}_{C V}=E[g]+\frac{1}{N} \sum_{1}^{N}\left(f_{i}-g_{i}\right)
$$

where the function $g$ is chosen so that it closely approximates $f$ and, moreover, permits a closed-form determination of $E[g]$. For direct Monte Carlo, the error variance is

$$
\sigma_{M C}^{2}=\sigma^{2} / N \triangleq \sigma_{D}^{2}
$$

where $\sigma^{2} \triangleq \operatorname{Var}[f]=E\left[f^{2}\right]-(E[f])^{2}$. For the control variate estimate, the corresponding Monte Carlo error variance is

$$
\sigma_{M C}^{2}=\sigma_{C V}^{2} / N
$$

with $\sigma_{C V}^{2}=\operatorname{Var}[f-g]$. For a suitably chosen approximating function $g$, we have $\sigma_{C V}^{2}<\sigma^{2}$, yielding variance reduction.

The fundamental problem of the control variate method is then how to determine a suitable approximating function $g$. This is especially true for problems of high dimensionality (i.e., multi-parameters). Following Chorin [2], we assume the general function space expansion 


$$
g=\sum_{0}^{m} a_{i} \Phi_{i}(\mathbf{x})
$$

where $\Phi_{i}(\mathbf{x})$ are orthonormal Hermite polynomials so that $E\left[\Phi_{i} \Phi_{j}\right]=\delta_{i j}$ and $\mathbf{x}$ is a Gauss vector of $p$ uncorrelated components. For $p=1$, we have

$$
\Phi_{0}=1, \Phi_{1}=x, \Phi_{2}=\left(x^{2}-1\right) / 2^{1 / 2}, \Phi_{3}=\left(x^{3}-3 x\right) / 6^{1 / 2}, \ldots,
$$

while for $p>1$, the general orthonormal Hermite polynomial is of the form $\Phi_{l_{1}}\left(x_{1}\right) \Phi_{l_{2}}\left(x_{2}\right) \cdots \Phi_{l_{p}}\left(x_{p}\right)$ with a restriction on the total order such that $l_{1}+l_{2}+\cdots+l_{p} \leq m$.

With these preliminaries, the basic Chorin estimator $f_{C}$ is given by Eq. (15) in [3] as

$$
f_{C}=\hat{f}-\Delta \hat{f}_{0}=\frac{1}{M} \sum_{i=1}^{M}\left\{f\left(\mathbf{x}_{i}\right)-\sum_{k=1}^{m} \hat{a}_{k}^{\prime} \Phi_{k}\left(\mathbf{x}_{i}\right)\right\}
$$

where $\Delta f_{0}$ is an estimate of the residual $f-E[f], \hat{a}_{k}^{\prime}$ are coefficient estimates to be precisely defined below and $m=m(m, p)=(m+p) ! / m ! p !$ is the series truncation point defined by the order restriction $m$ and the number of parameters $p$. The "corrector series" $\Delta f_{0}$ is then a zero-mean multi-dimensional Hermite polynomial expansion modeling the stochastic fluctuations in $f$ resulting from the random inputs $\mathbf{x}_{i}$.

The estimator $f_{C}$ is obtained by first dividing $N=2 M$ statistically independent samples into two sets

$$
\begin{aligned}
S & =\left\{\mathbf{x}_{i}, i=1,2, \ldots, M\right\} \\
S^{\prime} & =\left\{\mathbf{x}_{i}^{\prime}, i=1,2, \ldots, M\right\}
\end{aligned}
$$

and then using the set $S^{\prime}$ to estimate the coefficients

$$
\hat{a}_{k}^{\prime}=\frac{1}{M} \sum_{i=1}^{M} f\left(\mathbf{x}_{i}^{\prime}\right) \Phi_{k}\left(\mathbf{x}_{i}^{\prime}\right)
$$

by ordinary Monte Carlo. Next, the set $S$ is used to form the final estimate $f_{C}=f_{C}\left(S, S^{\prime}\right)$ given by Eq. (8) above. As shown in [3], the exact Monte Carlo error variance for the Chorin estimator is

$$
\sigma_{C}^{2}=2 A N^{-1}+4 B N^{-2}
$$

where

$$
A=\sigma^{2}-\sum_{1}^{m} a_{k}^{2}, \quad B=\sum_{1}^{m} \operatorname{Var}\left[a_{k}\right] .
$$

The quantity $A$, which is the dominant term for large $N$, is sometimes referred to as the mean square remainder $R_{m}$ where, by orthogonality,

$$
R_{m}=A=\sum_{m+1}^{\infty} a_{k}^{2}
$$

Thus we see how the corrector series leads to variance reduction.

In order to make better use of the available samples, two symmetrized estimators have been introduced $[3,4,5]$. By reversing the roles of the sample sets $S$ and $S^{\prime}$ and repeating the previous two-step procedure, two Chorin estimators 


$$
f_{1}=f_{C}\left(S, S^{\prime}\right), \quad f_{2}=f_{C}\left(S^{\prime}, S\right)
$$

are obtained. Their average then yields the symmetric Chorin $(S C)$ estimate

$$
f_{S C}=\frac{1}{2}\left(f_{1}+f_{2}\right)
$$

with the corresponding Monte Carlo error variance [3]

$$
\sigma_{S C}^{2}=A N^{-1}+2(B+C) N^{-2} .
$$

As a result of using all the available samples more effectively, the factor of 2 in the leading term of Eq. (11) has now been eliminated. However, an additional second-order penalty term

$$
C=\sum_{1}^{m} \sum_{1}^{m} E\left[f \Phi_{j} \Phi_{k}\right]
$$

has arisen.

Searching for further improvements, we next partitioned all $N$ Monte Carlo samples into three statistically independent sets of $M$ samples each $(N=3 M$ now $)$. Adding the set

$$
S^{\prime \prime}=\left\{\mathbf{x}_{i}^{\prime \prime}, i=1,2, \ldots, M\right\}
$$

to $S$ and $S^{\prime}$, three Chorin estimates are formed:

$$
f_{1}=f_{C}\left(S, S^{\prime}\right), \quad f_{2}=f_{C}\left(S^{\prime}, S^{\prime \prime}\right), \quad f_{3}=f_{C}\left(S^{\prime \prime}, S\right),
$$

and their average then gives the trisymmetric Chorin (TSC) estimate

$$
f_{T S C}=\frac{1}{3}\left(f_{1}+f_{2}+f_{3}\right) .
$$

It is shown in detail in [5] that the three estimates $f_{i}$ in Eq. (21) are mutually uncorrelated so that the resulting Monte Carlo error variance

$$
\sigma_{T S C}^{2}=A N^{-1}+3 B N^{-2}
$$

has no additional penalty term. Comparing Eqs. (11) and (22), we see that the TSC estimate has a smaller error variance than the $C$ estimate under all conditions. Hence the TSC estimator is universally better than the $C$ estimator.

Adaptive procedures. In order to obtain the best possible results using these estimators, it is most important to assess which terms in the corrector series $\Delta f_{0}$ given in Eq. (8) yield a net variance decrease. This is especially true if the dimensionality $p$ is high so the Hermite series becomes rather large. In fact, it is noted that the upper limit $m$ for the orthonormal expansion of $\Delta f_{0}$ in Eq. (8) is actually of the form $m(m, p)=$ $(m+p) ! / m ! p !$. For example, if $m=4$ (so that linear, quadratic, cubic, and quartic terms are included) and $p=5, m(4,5)=126$.

The method we use for selecting which terms in $\Delta f_{0}$ are important is referred to as the adaptive series selection algorithm. The label "adaptive" is used because the corrector series is automatically "tailored" for each value of $N$ used in the computations. This algorithm will be described here for the fully symmetrized TSC estimator, but it has also been implemented for the other estimators $C$ and $S C$.

Consider the Monte Carlo error variance for the TSC estimator given by Eq. (22) where $A$ is the mean square remainder in the truncated Hermite series expansion and $B$ is the sum of the error variances for the coefficient estimates $\hat{a}_{k}$. Inserting Eqs. (12) and 
(13) into (22), we have

$$
\sigma_{T S C}^{2}=\left[\sigma^{2}-\sum_{1}^{m}\left(a_{k}^{2}-M^{-1} \operatorname{Var}\left[a_{k}\right]\right)\right] N^{-1}
$$

since $N=3 M$. Then we define

$$
\Delta_{k} \triangleq a_{k}^{2}-M^{-1} \operatorname{Var}\left[a_{k}\right]
$$

and we see that variance reduction occurs for the $k$ th term provided $\Delta_{k}>0$. Since individual terms can now be assessed, the quantity $\Delta_{k}$ is called the variance reduction discriminant. Finally, since both terms in Eq. (24) are positive, it is more convenient to work with the normalized variance reduction discriminant defined by

$$
\bar{\Delta}_{k} \triangleq M^{-1} \operatorname{Var}\left[a_{k}\right] / a_{k}^{2} .
$$

For variance reduction, $0<\bar{\Delta}_{k}<1$.

Since the numerator is simply the error variance for the $M$ - sample estimate of the coefficient $a_{k}$, while the denominator is simply the magnitude squared of the coefficient estimate, it is seen that $\bar{\Delta}_{k}$ represents the inverse signal-to-noise ratio squared, $(S / N)^{-2}$. Hence we define the adaptive series selection criterion (tolerance) as

$$
\mathrm{Tol}=(S / N)^{-2}
$$

and require

$$
\bar{\Delta}_{k}<\text { Tol } \leq 1 .
$$

A further modification has recently been incorporated. For the smallest value of $N(6$, 12 , or some multiple of 6$)^{1}$, we begin the computation with $\mathrm{Tol}=\mathrm{Tol}_{0}(=1$ usually). The number of terms $n_{t}$ retained in the series, i.e. passing the requirement given by Eq. (27), is determined where, in general, $n_{t}<m(m, p)$. Then $N$ is increased and all computations are repeated. If $n_{t}$ is now greater than the previous value, the tolerance is set equal to the closest available value of $\bar{\Delta}_{k}$ which is smaller than the present value of Tol. If $n_{t}$ is less or equal to the previous value, the present value for Tol is retained. In this way, the accept/reject criterion for the adaptive series selection is continuously adjusted as the sample size $N$ is increased. This refinement has been found to be especially valuable for computations involving a large number of parameters $(p \leq 40)$.

Bullet model problem. In order to illustrate the operation of this adaptive Monte Carlo algorithm, a simple one-dimensional model problem with five random perturbation variables is treated in detail in this section. This so-called "bullet problem" [3] is defined by the differential equations

$$
\dot{x}=\alpha v+\beta, \quad \dot{v}=\gamma x+\delta v+\varepsilon
$$

and the nominal $(\beta=\varepsilon=0)$ initial conditions $x_{0}$ and $v_{0}$. Each of the five coefficients $\alpha, \beta$, $\gamma, \delta, \varepsilon$ is non-time-varying but is assumed to have the form

$$
\rho=\rho_{0}+\rho^{\prime}
$$

\footnotetext{
${ }^{1}$ Results are routinely obtained for all three estimators $C, S C$, and $T S C$; consequently, each sample set $N$ must be divisible by both 2 and 3 .
} 
with a nominal value $\rho_{0}$ and a random component $\rho^{\prime}$. If we set

$$
\zeta \triangleq\left(\delta^{2}+4 \alpha \gamma\right)^{1 / 2}
$$

and

$$
r_{1}, r_{2}=\frac{1}{2}[\delta \pm \zeta]=-\tau_{1}^{-1},-\tau_{2}^{-1}
$$

for the two "time constants" $\tau_{i}$, the solution of $(28)$ is

$$
x(t)=C_{1} e^{r_{1} t}+C_{2} e^{r_{2} t}+\frac{\alpha \varepsilon-\delta \beta}{2 \alpha \gamma}\left[e^{r_{1} t}+e^{r_{2} t}-2\right]
$$

with

$$
C_{1} \triangleq \frac{1}{2}\left(1-\frac{\delta}{\zeta}\right) x_{0}+\frac{\alpha}{\zeta} v_{0}, \quad C_{2} \triangleq \frac{1}{2}\left(1+\frac{\delta}{\zeta}\right) x_{0}-\frac{\alpha}{\zeta} v_{0}
$$

where $x_{0}$ and $v_{0}=(1 / \alpha) \dot{x}(0)$ are the nominal initial conditions at $t=0$ with both random noise terms $\beta$ and $\varepsilon$ equal to zero. Also, using Eq. (28),

$$
v(t)=\frac{1}{\alpha}[\dot{x}(t)-\beta]
$$

while $\dot{x}(t)$ is immediately computable from Eq. (31).

For the simulations, the following nominal values:

$$
\alpha_{0}=1.0, \quad \beta_{0}=0.0, \quad \gamma_{0}=-0.8, \quad \delta_{0}=-2.4, \quad \varepsilon_{0}=0.0,
$$

were chosen for the five parameters. The corresponding time constants are then $\tau_{1}=2.5$, $\tau_{2}=0.5$. Next, we wish to allow some variability in the parameter set $(\alpha, \beta, \gamma, \delta, \varepsilon)$ while maintaining the inequalities

$$
\alpha>0, \quad \delta<0, \quad-\delta^{2} / 4 \alpha<\gamma<0,
$$

so that the solution $x(t), v(t)$ is indeed given by two decaying exponentials.

By using the equidistributed random number generator described previously [3, p. 356], so-called normal zero-one $N(0,1)$ Gaussian random numbers $\xi$ were produced with a theoretical mean of zero and variance of one. Hence, in order to maintain reasonably small perturbations about the nominal trajectory and to guarantee that $\zeta^{2}>0$ "almost always", the following scaling values $s_{\rho}$ :

$$
s_{x}=0.05 \quad s_{\beta}=0.10 \quad s_{\gamma}=0.08 \quad s_{\delta}=0.07 \quad s_{\varepsilon}=0.10
$$

were introduced for each of the five parameters so the random components are then given by

$$
\rho^{\prime}=s_{\rho} \xi
$$

The following "standard" case:

$$
x_{0}=6.0, v_{0}=-2.0, t_{f}=\text { final time }=2 \tau_{1}=5.0
$$

was chosen. Figure 3 in [3] shows the nominal and four perturbed trajectories for this case. For additional information, the reader is referred to [3].

A so-called impact dispersion footprint is shown in Fig. 1 for the standard case 
DISPERSION FOOTPRINT

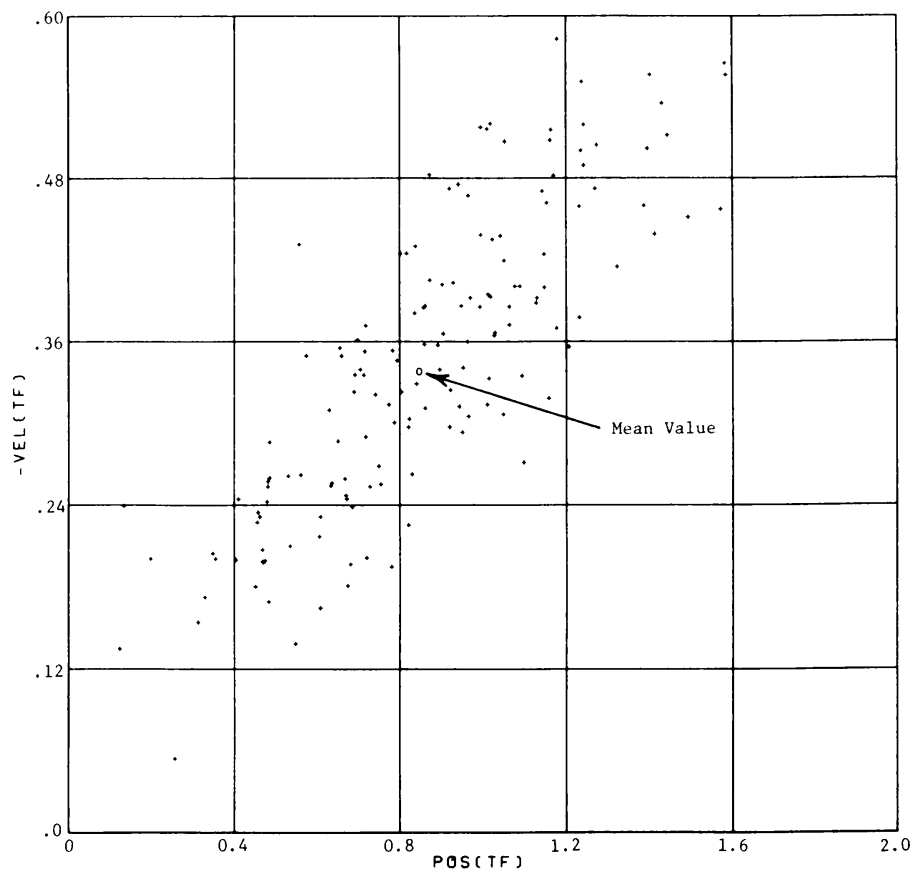

Fig. 1. Dispersion footprint for the "bullet" example problem; 150 impact points are plotted.

defined by Eq. (35). Note that the final time $t_{f}$ is different for each perturbed trajectory by virtue of Eqs. (29) and (30).

Simulation results using the adaptive symmetrized estimators are presented next in Figs. 2 and 3 for the position estimates and in Figs. 4 and 5 for the velocity estimates. The various estimates of the mean are given in the first figure of each set while the corresponding Monte Carlo error variances are shown in the second figure of each set. For these results, a linear fit $(m=1)$ to the corrector series was used so the maximum series length is $m(1,5)=6$. Also, since the basic Chorin estimator has been shown previously [5] to perform quite poorly relative to the symmetrized estimators $S C$ and $T S C$, results obtained with the $C$ estimator have been eliminated in Figs. 2 to 5 .

The acceleration towards convergence is highly visible in these figures. As $N$ is increased, both advanced estimators $S C$ and TSC yield highly stabilized and consistent results for the mean values $\bar{x}\left(t_{f}\right)$ and $\bar{v}\left(t_{f}\right)$. However, the major payoff is shown in Figs. 3 and 5 where the Monte Carlo error variances are plotted as a function of $N$. These latter figures can be interpreted in either of two ways. For a given $N$, the reduction in variance is immediately available while, for a given variance, the allowable reduction in sample size $N$ is easily determined. Table 1 gives, for several values of $N$, further data on the resultant series selected by the adaptive algorithm, the tolerance level chosen by the simulation and the variance reduction achieved. Finally, we note that negative variances are not plotted in Figs. 3 and 5 (hence the visible gaps). This occurs, especially for small values of $N$, as a result of poor estimation of both the variance $\sigma^{2}$ and the coefficients $a_{k}$ so that the difference specified by Eq. (12) is negative. 
ONE-DIM MONTE CARLO SIMULATION $(M=1$. TOL $0=1.0)$

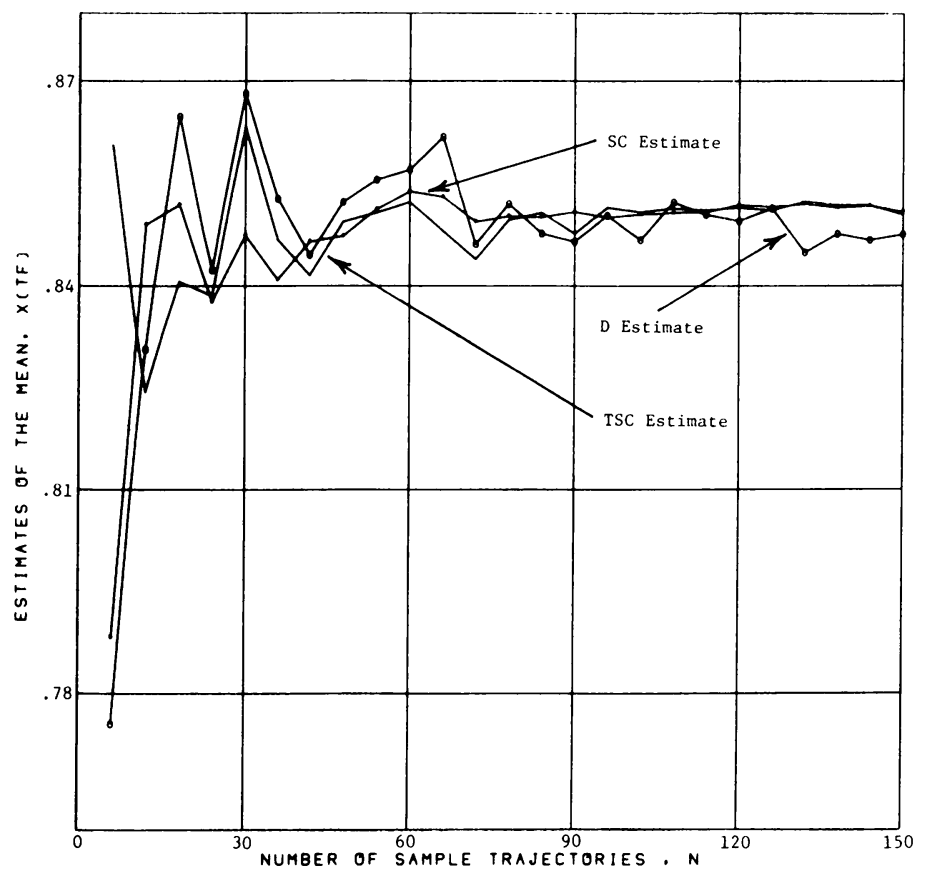

FIG. 2. Three estimates for the mean position at the final time $x\left(t_{f}\right)$. Here, as in all the following plots, the symbols represent-॰-Direct estimate $D, *$-Symmetrized Chorin estimate $S C$, - - Trisymmetric Chorin estimate $T S C$.

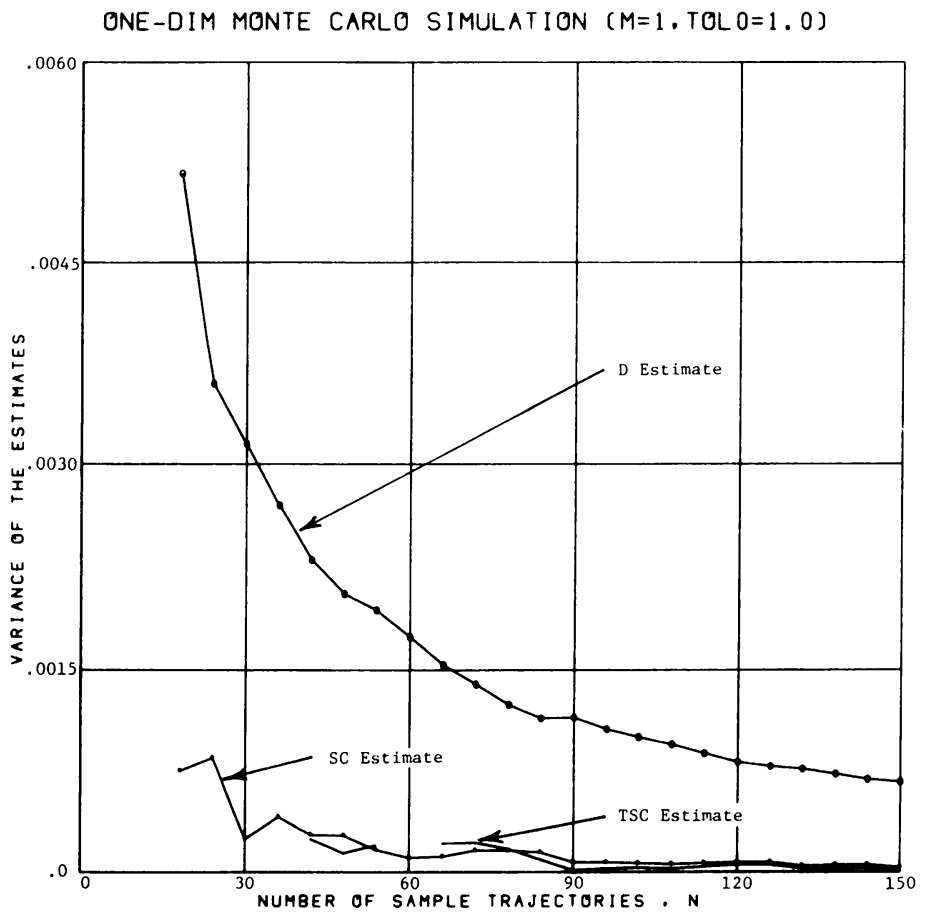

FIG. 3. Monte Carlo error variances for the three estimates shown in Fig. 2. Note the reduction in variance obtained with the symmetrized adaptive estimators compared to the ordinary Monte Carlo variance $\sigma_{D}^{2}$. 
ONE-DIM MONTE CARLO SIMULATION $(M=1$, TOL $0=1,0)$

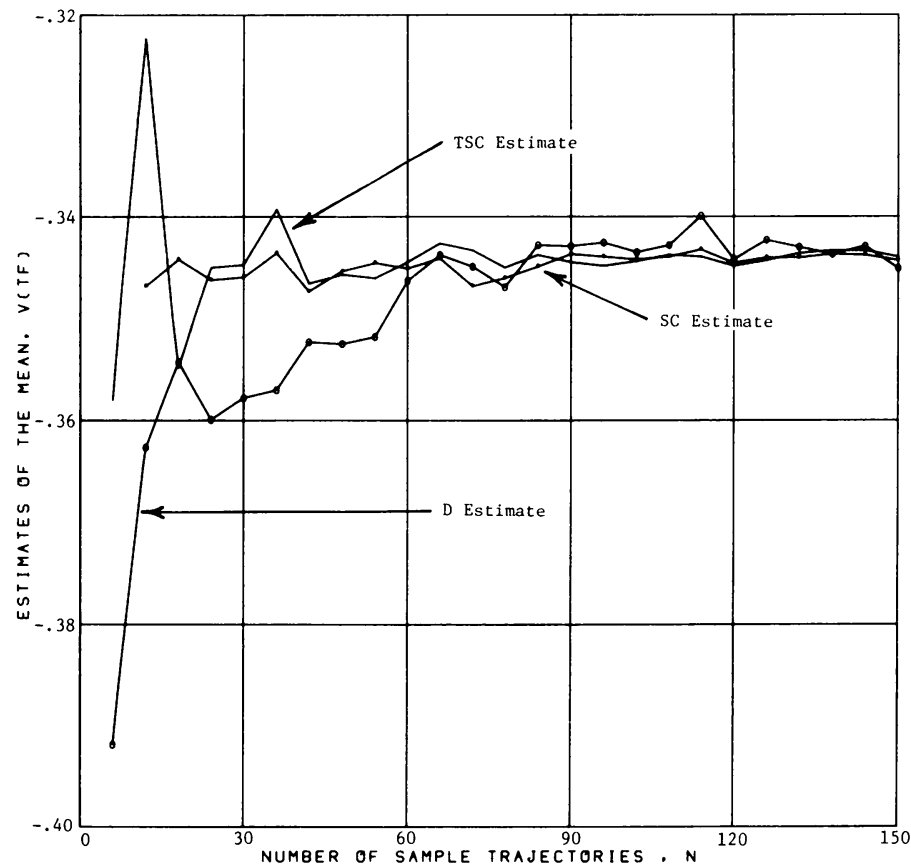

Fig. 4. Three estimates for the mean velocity at the final time $v\left(t_{f}\right)$.

ONE-DIM MONTE CARLO SIMULATION $(M=1$, TOL $0=1.0)$

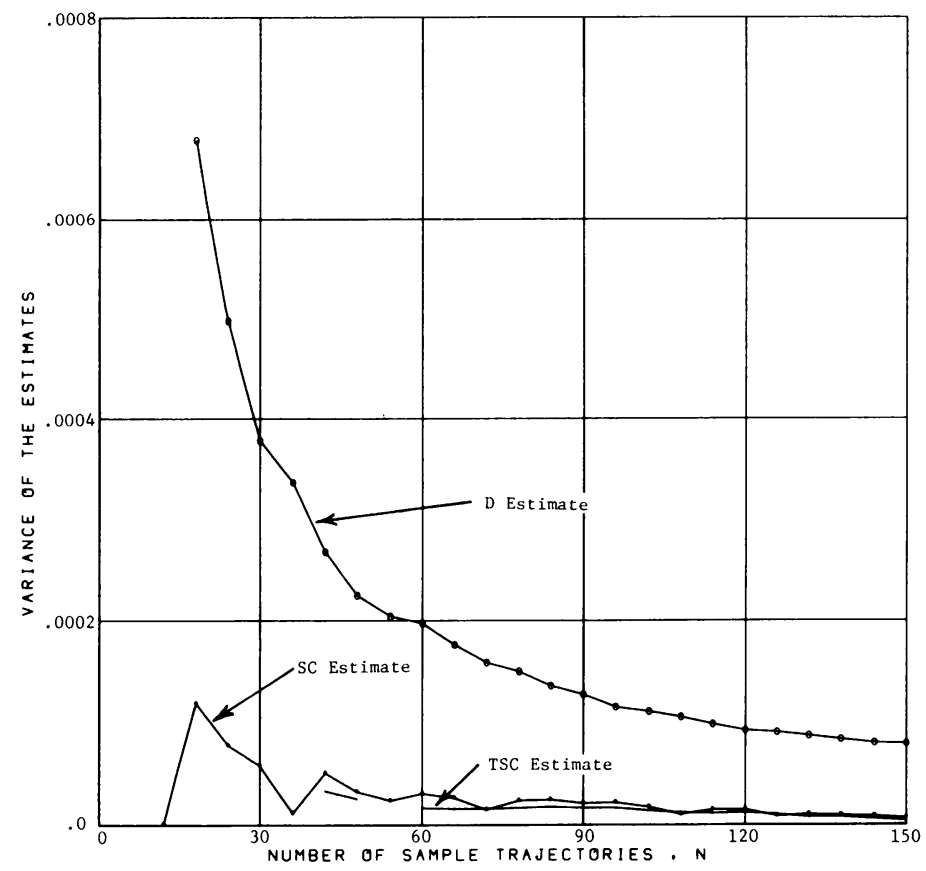

Fig. 5. Error variances for the three estimates shown in Fig. 4. 
TABLE 1. Performance of the adaptive series selection algorithm with $m=1$ and $\mathrm{Tol}_{0}=1$.

\begin{tabular}{c|r|c|c|c}
\hline \multirow{2}{*}{ Quantity } & \multicolumn{1}{|c|}{$N$} & Tol & Parameters & Variance Reduction Ratio \\
& \multicolumn{1}{|c|}{$\sigma_{D}^{2} / \sigma_{S C}^{2} \quad$ or $\sigma_{D}^{2} / \sigma_{T S C}^{2}$} \\
\hline$S C$ Position Estimate & 60 & 0.39784 & $\beta \varepsilon$ & 17.11 \\
& 120 & 0.39784 & same & 11.04 \\
& 150 & 0.39784 & same & 18.45 \\
TSC Position Estimate & 60 & 0.32623 & $\beta \varepsilon$ & $\left(\sigma_{T S C}^{2}<0\right)$ \\
& 120 & 0.30668 & same & 15.52 \\
& 150 & 0.30668 & same & 47.87 \\
\hline SC Velocity Estimate & 60 & 0.49911 & $\beta \gamma$ & 6.37 \\
& 120 & 0.28492 & same & 6.28 \\
& 150 & 0.28254 & same & 11.73 \\
TSC Velocity Estimate & 60 & 0.37187 & $\beta \gamma$ & 12.11 \\
& 120 & 0.37187 & same & 7.57 \\
& 150 & 0.37187 & same & 18.30 \\
\hline
\end{tabular}

Evaluation of stochastic error model. The key factor in this variance reduction procedure is the identification, during the computation, of an approximate stochastic system model (the "corrector series" $\Delta f_{0}$ in Eq. (8)). This Hermite polynomial expansion attempts to model, in the sense of minimum mean square error [3,4], the stochastic fluctuations in $f$ resulting from the random inputs $\mathbf{x}_{i}$.

In this section, the accuracy of these approximate error models will be evaluated, for a specified value of $N$, as a function of the adaptive tolerance level Tol. With an expansion containing only linear terms $(m=1)$, the stochastic error model $g_{i}^{Q}=g^{Q}\left(\mathbf{x}_{i}\right)$ is obtained for each of the estimates $Q=C, S C$, or TSC. This calculation is performed for the following values of the tolerance:

$$
\mathrm{Tol}=0,0.05,0.1, \ldots, 1.0
$$

in the unit interval. Note that, when Tol $=0$, no terms are retained in the $g_{i}^{Q}$ series, so that the resulting estimates simply revert to the standard (or direct $D$ ) Monte Carlo values. On the other hand, as shown by Eqs. (24) and (27), Tol $\leq 1$ for variance reduction. Once the $g_{i}^{Q}$ have been obtained, these functions are evaluated for each of the $N$ sample trajectories and the rms error $\varepsilon_{Q}$ defined by

$$
\varepsilon_{Q} \triangleq\left|N^{-1} \sum_{i=1}^{N}\left[f\left(\mathbf{x}_{i}\right)-g^{Q}\left(\mathbf{x}_{i}\right)\right]\right|^{1 / 2}
$$

is computed.

The results of this calculation for the bullet problem are given in Figs. 6 and 7 for the position and velocity estimates, respectively, at the final time. Beginning with Tol $=0$ in Fig. 6 for the position estimates, the first (and largest) reduction in $\varepsilon_{Q}$ occurs when the $\beta$ term is retained while the second reduction occurs when the term for $\varepsilon$ is also included. In a similar fashion, the velocity error model first includes a $\beta$ term, then a $\gamma$ term and, finally, a $\delta$ term as the tolerance level is progressively increased.

Numerical partial derivatives have been computed [3, p. 362]. It is most interesting that the largest partial derivatives (in absolute value) are:

$$
\frac{\partial x\left(t_{f}\right)}{\partial \beta}=2.79692, \quad \frac{\partial x\left(t_{f}\right)}{\partial \varepsilon}=1.16538
$$


ONE-DIM ERROR MODEL EVALUATION $(M=1, N=60)$

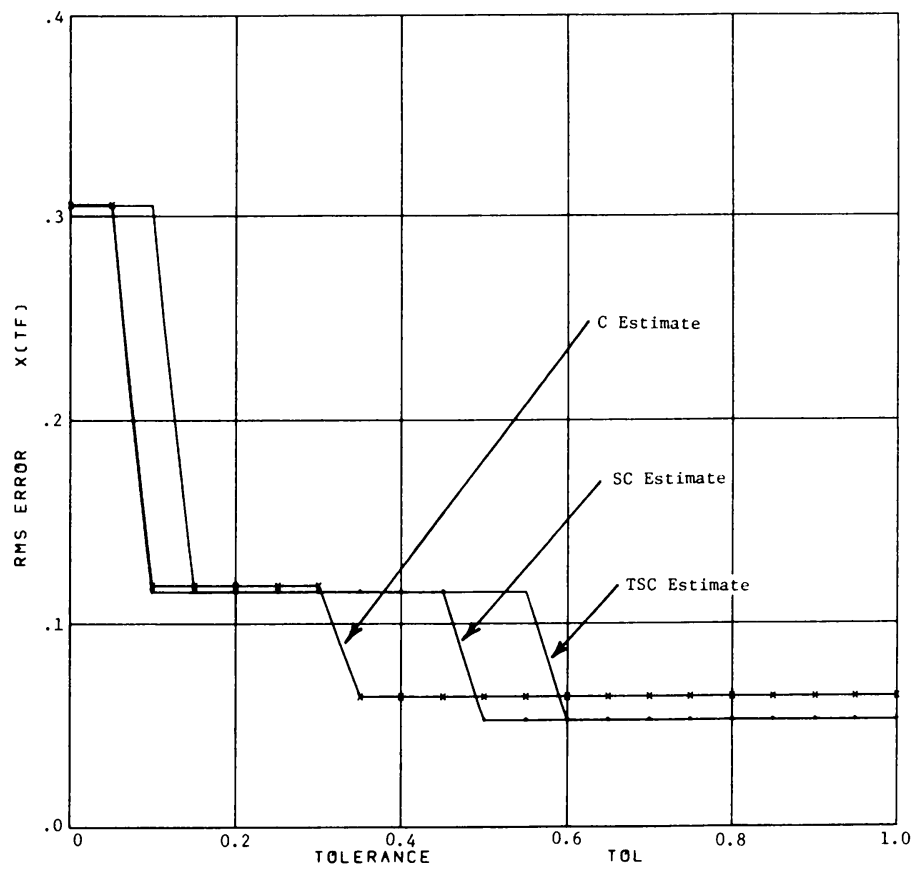

Fig. 6. Resulting rms position error as a function of the tolerance level for a linear correction series of five terms and a total of 60 trajectories. Here $x$ denotes the original Chorin estimator. The stochastic series yields about a factor of 6 reduction in the rms error.

ONE-DIM ERROR MODEL EVALUATION $(M=1, N=60)$

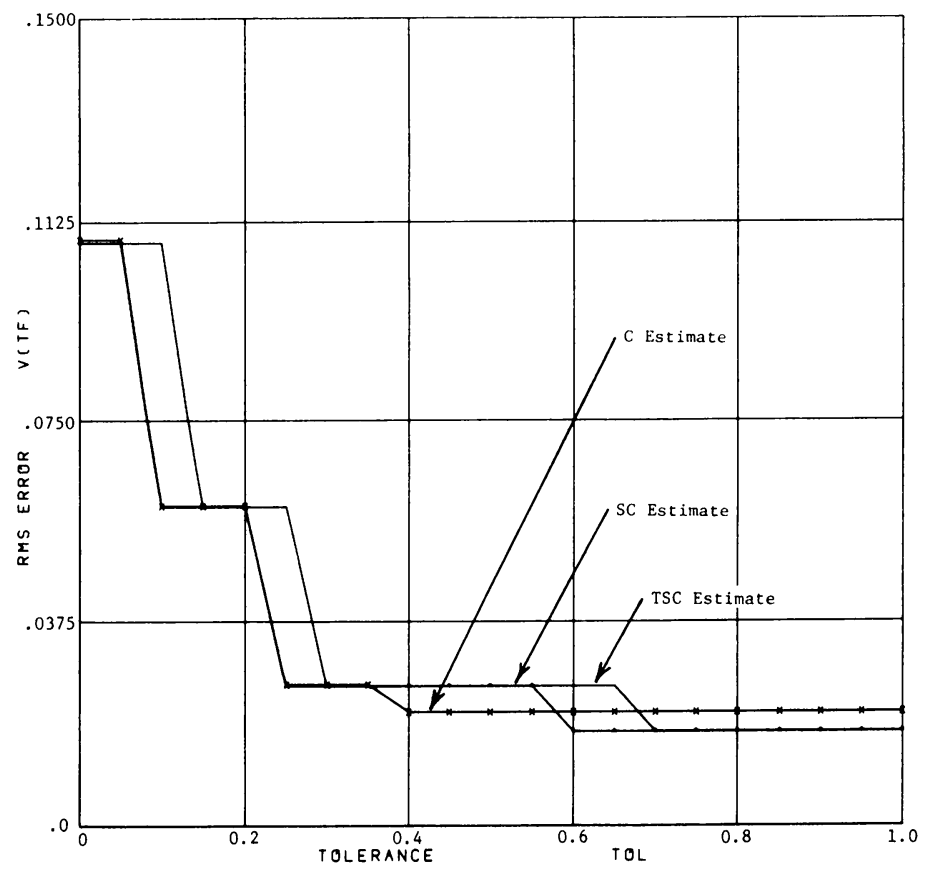

FIG. 7. Resulting rms velocity error as a function of the tolerance level. The stochastic series gives roughly a factor of 5 improvement in the rms error. 
and

$$
\frac{\partial v\left(t_{f}\right)}{\partial \beta}=-0.91866, \quad \frac{\partial v\left(t_{f}\right)}{\partial \gamma}=0.66545, \quad \frac{\partial v\left(t_{f}\right)}{\partial \delta}=-0.27451 .
$$

Also, the generally inferior behavior of the basic Chorin estimator $C$ is confirmed again in these two figures. Finally, we want to note that, for a problem with very high dimensionality $(p=40)$, the $r m s$ error curves $\varepsilon_{Q}$ (Tol) assumed a much more parabolic shape, with strong minima occurring for values of Tol inside the available interval $0 \leq \mathrm{Tol} \leq 1$.

Conclusions. As shown both in theory and in numerical experiments, the symmetrized estimators $S C$ and $T S C$ have been able to furnish valuable reductions in Monte Carlo estimation errors. Furthermore, the adaptive series selection technique has proven to be an essential addition to these advanced Monte Carlo estimators. The adaptive algorithm is based on the fact that the variance reducing (or increasing!) effect of each term in the complete (and generally nonlinear) correction series can be isolated. This important result is obtained by inspection of the exact theoretical expression for the estimator variance and leads to the so-called variance reduction discriminant given by Eq. (24).

We consider the use of these orthonormal Hermite expansions to be a definite advance in Monte Carlo computations since, in general, both variance reduction and system identification are provided. In particular, the further insights obtained from the additional processing of the basic trajectory data should prove, in most cases, to be of considerable importance. Since it is hoped that others might apply these techniques to their problems, the current version of the computer program has been fully documented [6].

\section{REFERENCES}

[1] W. Freiberger and U. Grenander, A short course in computational probability and statistics, Springer-Verlag, New York, 1971

[2] A. J. Chorin, Hermite expansions in Monte Carlo computation, J. Comp. Phys. 8, 472-482 (1971)

[3] F. H. Maltz and D. L. Hitzl, Variance reduction in Monte Carlo computations using multi-dimensional Hermite polynomials, J. Comp. Phys. 32, 345-376 (1979)

[4] F. H. Maltz and D. L. Hitzl, Nonlinear adaptive estimation procedures for increasing the efficiency in Monte Carlo computations, J. Guid. and Cont. 3, 251-256 (1980)

[5] D. L. Hitzl and F. H. Maltz, Adaptive estimation procedures for multi-parameter Monte Carlo computations, J. Comp. Phys. 37, 218-241 (1980)

[6] F. Zele and D. L. Hitzl, Advanced Monte Carlo software for IAP applications, LMSC technical report D676956, 1979 